\title{
Functional and Structural Investigation of Songbird Brain Projection Neurons with Shuttle and Find
}

\author{
Michelle Husain, ${ }^{*}$ Dr. Christian Thomas, ${ }^{* *}$ Moritz Kirschmann, ${ }^{* * *}$ Daniele Oberti, ${ }^{* * * P r o f .}$ \\ Dr.Richard Hahnloser \\ * Carl Zeiss NTS, 1 Corporation Way, Peabody MA 01960 husain@nts.zeiss.com \\ ** Carl Zeiss AG Corporate Research and Technology Oberkochen, Deutschland. \\ *** Institute of Neuroinformatics, University of Zurich and ETH Zurich, Switzerland
}

The singing behavior of songbirds and human language share many similarities. As language in humans, the song of the bird is not innate but learned from a tutor. Therefore songbirds are a good animal model to study brain areas and networks involved in this complex behavior. Similar brain structures in birds and humans are thought to steer these learning processes, as human language and birds singing are related processes. Therefore the understanding of learning processes in a songbird brain is a step towards understanding the learning and speech of language in humans. Within a songbird brain several regions are involved in song learning and production as shown in Fig. 1. Several brain areas are connected to the High Vocal Center(HVC) region which is the main premotor area for vocal production. In this application connections within HVC are analyzed on an ultrastructural level. Cells projecting to the brain region projecting neurons are labeled with a tracer and their connectivity within HVC can be investigated. Correlative Light and Electron Microscopy (CLEM) is essential for this application since both the identification of the projection cells in the HVC region via Fluorescence Light Microscopy (FLM) and ultrastructure of the same cells in Scanning Electron Microscopy (SEM) are necessary.

The sample was prepared in a similar manner to the protocol described in detail in [1]. A specific region with neuron density of a zebra finch brain was located by stereotaxic coordinates and injected with $0.5 \mu$ Alexa 488 dextran. The area was then prepared for SEM imaging by perfusion, fixation, dehydration, and embedding for sectioning. 60-90nm sections were transferred onto Indium Tin coated coverslips. This coating reduces charging in SEM and a high optical transparency is still assured.

The coverslip was placed into the sample holder especially designed for CLEM by Carl Zeiss. This holder can be used in Light Microscope as well as Scanning Electron Microscopy so that the sample is stably fixed in the holder during the whole imaging process. The holder has three fiducial markers which define a coordinate system that can be calibrated very fast and semiautomatically in the Shuttle \& Find module of the AxioVision Software. FLM of the sections was performed with an Axio Observer.Z1 using a 100 x objective (EC Epiplan-Neofluar 100 x / 0.90 HD DIC) and a filter set with 470/40 nm excitation and $525 / 50 \mathrm{~nm}$ emission. The microscope was equipped with an AxioCam HRm. Regions of interest (ROIs) were defined and selected in the fluorescence image. Next the sample was transferred to a SUPRA 40VP SEM. The holder was calibrated semiautomatically and after a few seconds the frame imaged in the FLM was located. A subsequent fine calibration allows imaging the selected ROIs at a precision below $5 \mu \mathrm{m}$. SEM imaging was done at an acceleration voltage of $1.5 \mathrm{kV}$ with the in-lens secondary electron detector. 
Fluorescent spots indicate cell compartments where the tracer is localized in HVC area in Fig 2. A neuron projecting from $\mathrm{HVC}$ is selected as ROI and then imaged in the SEM as shown in Fig 3. The SEM image has a pixel size of $4.6 \mathrm{~nm}$ which is sufficient for this application as it clearly resolves the ultrastructure of subcellular structures in the neuron as mitochondria, myelinated axons or synaptic vesicles. They are labeled by fluorescent tracers and imaged in FLM as well as in SEM. The overlay allows for classification of neurons observed in the SEM based on information from LM. SEM imaging of the same sections gives ultra structure information, e.g. presence of active synapses.

\section{References}

[1] D. Oberti, M.A. Kirschmann and R.H. Hahnloser Front. Neuroanat. 4:24 (2010) doi:10.3389/ fnana.2010.00024
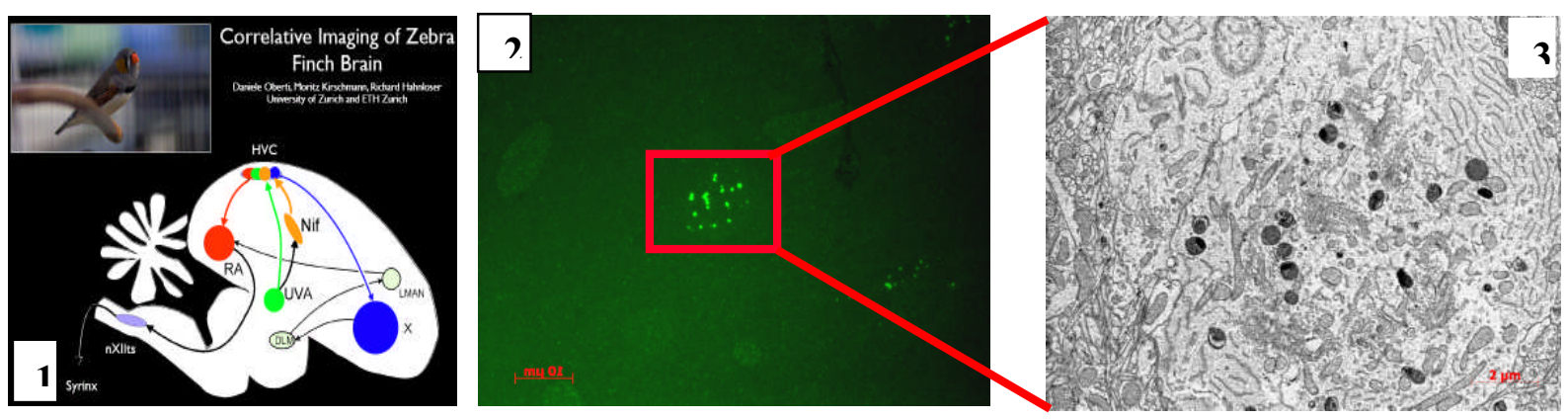

Fig. 1. Regions of Zebra Finch Brain involving song learning.

Fig. 2. Widefield Light Microscope image displaying flouresently labelled neuron in High Vocal Center of Songbird.

Fig. 3. SEM image of ROI from LM image with in-lens secondary electron detector at $1.5 \mathrm{kV}$ accelerating voltage.

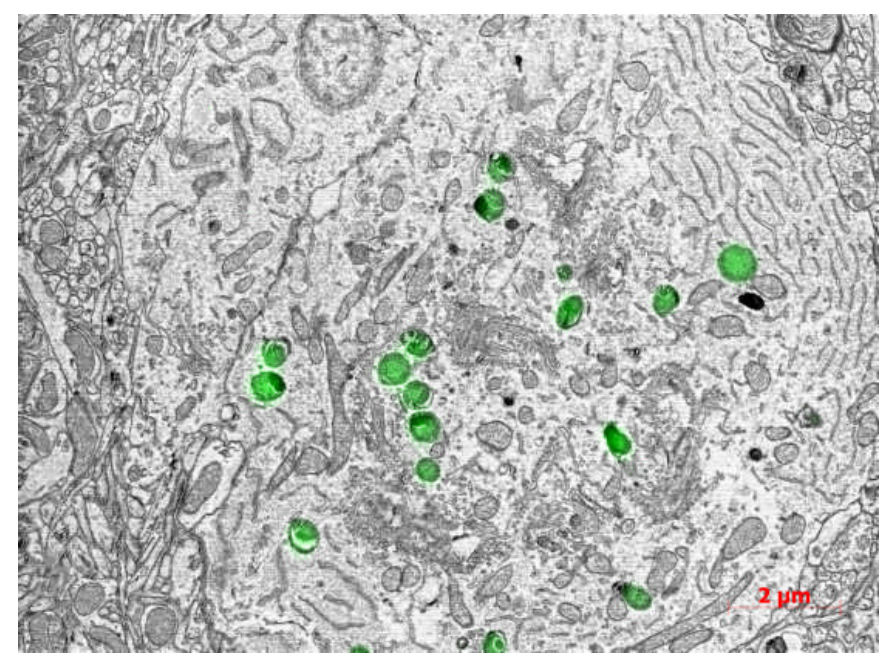

Fig. 4. Ovelay of ROI from both LM \& EM, combining fluorescent and ultrastructural information. 